\title{
A Convenient Synthesis and Spectral Studies of Diamines
}

\section{Derivatives}

\author{
Sudershan R. Gondi*, Michael B. Jacobsson, Christiana Julia Rissing and David Y. Son \\ Department of Chemistry, Southern Methodist University, \\ Dallas, Texas, USA
}

\begin{abstract}
A new series of substituted anthranilic esters derivatives linked with a 1,3-dithiolane and benzyloximino moiety was synthesized using the simple esterfication reaction and products were fully characterized. The isolated yields of these compounds range from 59 to $96 \%$. 1,3dithiolane ester and the benzyloxy substituted diamine derivatives are white solids and stable to air and moisture. The synthesized compounds can be exhibits UV-vis absorption properties by their structures with a amine or amide group, It is observed that absorption maximum is excellent for 2,6-disubstituted benzyloxy esters which can be explained by electron transfer or conjugation is steric effect in ortho substitution from the amino group and the amide group.
\end{abstract}

Keywords: dithiolane; benzyloximates, diamines, supramolecular design, spectral studies

\section{INTRODUCTION}

In recent years, crystal engineering and construction of coordination networks with fascinating structural topologies have attracted great attention owing to their potential as functional materials. $^{1-2}$ Concurrently, the development of multidimensional networks based primarily on linking metal centers with rigid bridging components, such as $4,4^{\prime}$ bipyridine has been initiated. ${ }^{33}$ 
Since then chemiluminescence's of 3-aminophthalhydrazide was first investigated by Albrecht, ${ }^{4}$ the acyl hydrazides are growing interest in the development of luminescent materials due to their potential application in emissive devices. ${ }^{5}$ Moreover, the attractive and promising practical applications in many other areas have stimulated further investigations in light-emitting devices, nonlinear optics, and functional films, ${ }^{6}$ conjugated polymers, ${ }^{7}$ logic functions of molecularscale ${ }^{8}$ uranyl salts, saccharides, and aromatic organic molecular crystals which exhibit tribofluorescence or tribophosphorescence from the molecules comprising the crystal and/or nitrogen emission triboluminescence, ${ }^{9}$ azopolymers. ${ }^{10}$ Metal directed assembly have been used to generate luminescent materials ${ }^{11}$ based on complexation of transition metal and multifunctional bridging ligands and is one of many useful strategies to design extended frameworks of various topology and dimensionality. Ligands with amino group of anthranilic acid derivative backbones may affect the properties of the resulting emissions by promoting the coupling of metal atoms through its emission systems. It has been of interest to use photoactive ligands as building blocks to generate supramolecular polymers. Some of these workers have been concerned with the influence of structural changes upon the chemiluminescent properties. ${ }^{12-13}$

\section{PRESENT WORK}

The luminescence is appreciably enhanced when the hydroxyl or amine groups are in the ortho position. The efficiency of chromogenic sensing is more remarkably affected by the chemical environment of the anthranilic acid derivatives, depending on the presence of a protecting free amino group or amide group. Taking into account these considerations, it is possible to optimize

the uv-vis sensing of the molecules by structure modification. Keeping the above applications in mind, we have investigated the synthesis and characterization of a series of 2,6- and 2,3-diamino 
benzoic methyl esters derivatives with various electron-donor groups at the 2,6- or 2,3-positions. 3-nitropthalic acid and its anhydrides was chosen as a starting material due to its ready availability and to the fact that it can be easily converted to diamine derivative $\mathbf{5}$ and $\mathbf{1 0}$, a compound that can be selectively functionalized at the amino positions. The electron donor groups chosen for this study were pyridyl, 1,3-dithiolanyl, benzyloximate, and thiophenyl ether, all known for their Lewis basicity and ligating potential. This investigation reflects our ongoing interest in synthesizing new organic ligands for supramolecular design. ${ }^{14-21}$

The initial step is the functionalization described herein was the conversion of 3-nitropthalic anhydride and 3-nitropthalic acid to 2,6-diamino benzoic acid methyl ester (5) and 2,3-diamino benzoic acid methyl ester (10) respectively (scheme 1 and 2).

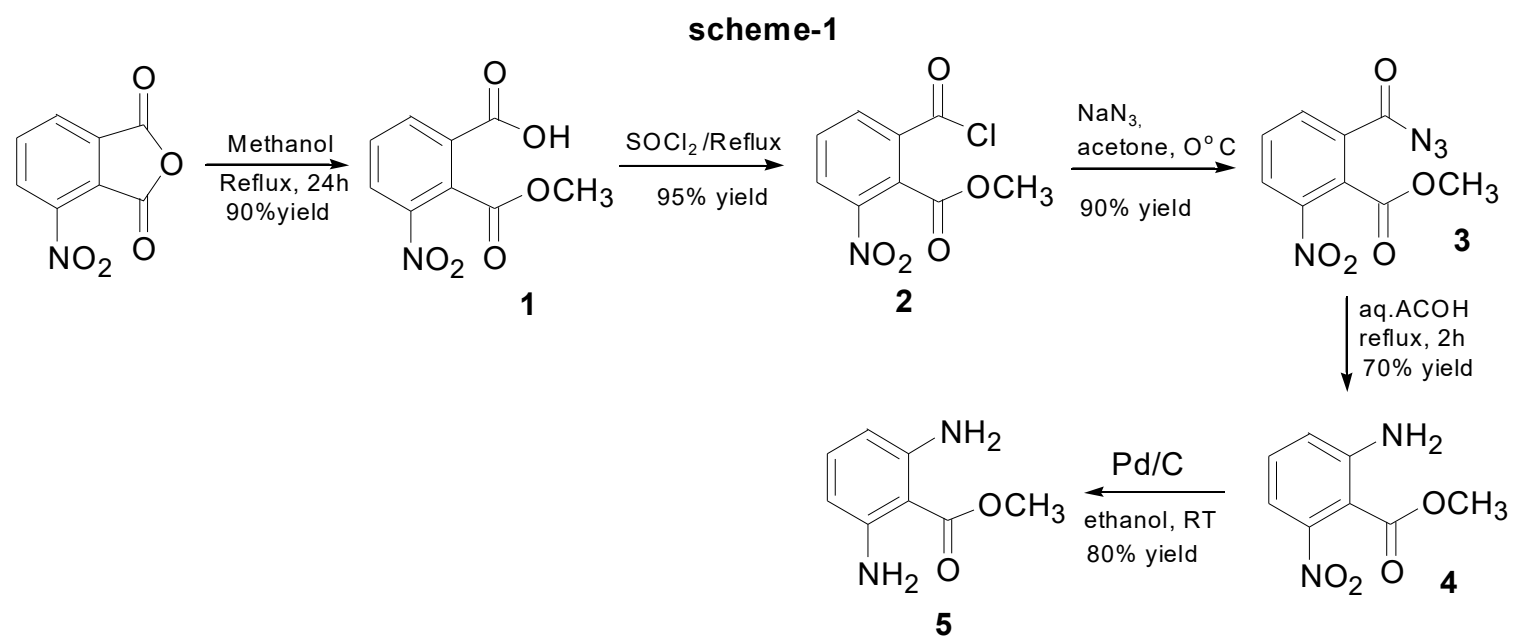

3-nitrophthalic anhydride under methanol reflux conditions gives mixture of $\mathbf{1}$ and $\mathbf{6}$ in ratio of 9:1 (by NMR) in 98\% yield. The mixture on treatment with thionyl chloride under heating condition gives corresponding mixture of acid chlorides $\mathbf{2}$ and $\mathbf{7}$ from which the acyl azide $\mathbf{3}$ and 8 were prepared by treating with sodium azide in acetone in $90 \%$. The mixture of acyl azides 3 and $\mathbf{8}$ under Curtius rearrangement followed by column chromatography purification gives two 
isolated product 4 and 9 in $70 \%$ and 5\% respectively. The compound 4 under palladium charcoal treatment gives product 5 in $90 \%$ yields.<smiles>O=C(O)c1cccc([N+](=O)[O-])c1C(=O)O</smiles><smiles></smiles><smiles>COC(=O)c1cccc([N+](=O)[O-])c1C(=O)O</smiles>

6<smiles>COC(=O)c1ccc([N+](=O)[O-])c([N+](=O)[O-])c1C(=O)Cl</smiles><smiles>N#[W]</smiles><smiles>COC(=O)c1cccc([N+](=O)[O-])c1C(N)=O</smiles>

8 aq.ACOH
reflux, $2 \mathrm{~h}$
$70 \%$ yield<smiles>COC(=O)c1cccc(N)c1N</smiles><smiles>COC(=O)c1cccc([N+](=O)[O-])c1N</smiles>

The compound 9 was independently prepared in four steps by regioselective esterfication of 3 nitro phthalic acid, followed by its acid chloride 7 in 95\% yield, which on acyl azide conversions 8 in $70 \%$ yield, followed by and Curties rearrangement. In these it is $100 \%$ regioselective conversion, no trace of its corresponding isomer was detected even in crude NMR. The compound 9 under reduction by using palladium charcoal condition gives the 2,3-diamino benzoic acid methyl ester 10 in $80 \%$ yield, and acetonide protected diamine $\mathbf{1 6}$ (in 5\%), is obtained as byproduct. The mechanism is unclear.

Derivatives 13-14 were each synthesized in straightforward two step procedures from 5 and 10 as summarized in Scheme 1. The diamines $\mathbf{5}$ and $\mathbf{1 0}$ on treatment with $\alpha$-bromo acetyl chloride to give the bromo derivatives 11 and 12 in $69 \%$ and $66 \%$ yields, respectively. The bromo derivatives $\mathbf{1 1}$ under treatment with 2-pyridyl methanol gives the pyridyl derivative $\mathbf{1 3}$ as an oil in $59 \%$ yield after chromatographic purification. Attempts to prepare $\mathbf{1 4}$ by reaction of $\mathbf{1 2}$ with 
2-pyridyl methanol in the presence of triethylamine or $\mathrm{NaH}$ were unsuccessful and resulted in the isolation of pyridyl ester product with elimination of aromatic moiety (by $\mathrm{nmr}$ ), it is understood that due to the steric hindrance, elimination of aromatic moiety might occur. (Scheme 3).
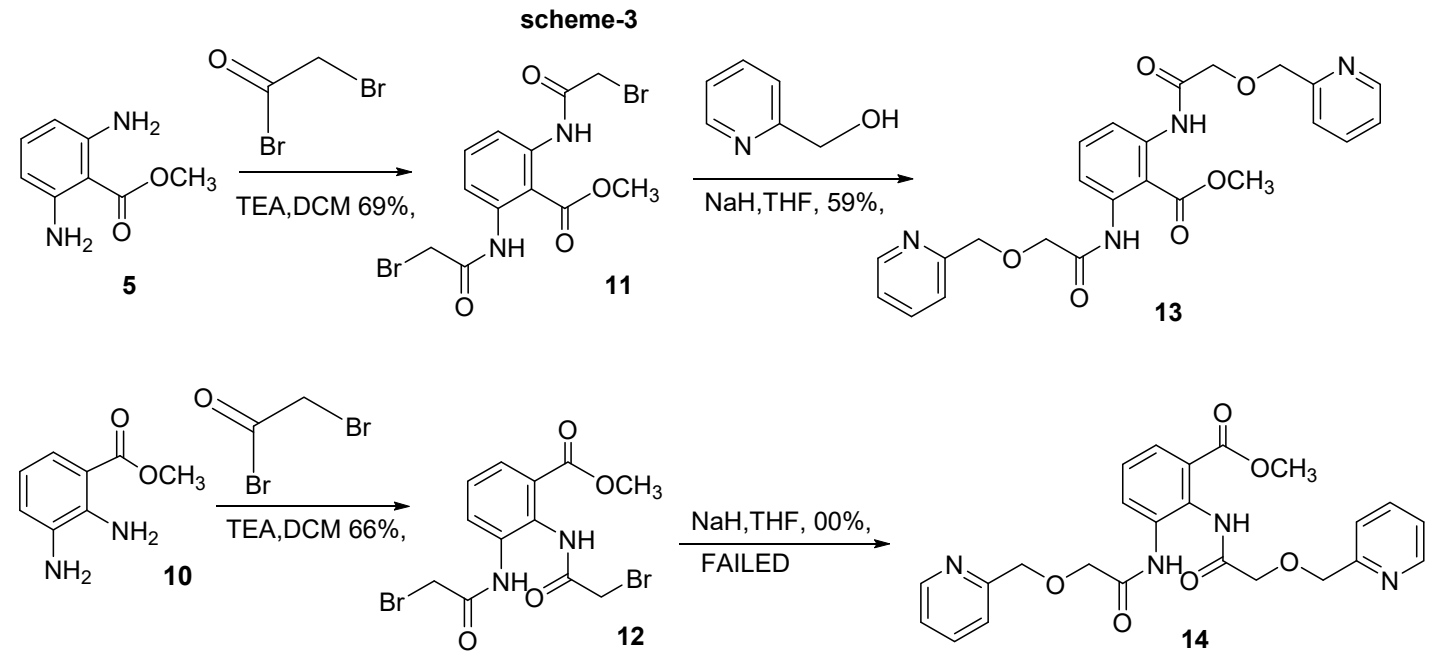

Reaction of $\mathbf{5}$ and $\mathbf{1 0}$ with 2.2 eq of 1,3-dithiolane-2-carbonyl chloride ${ }^{17}$ in the presence of triethylamine in THF led to the isolation of the bis(1,3-dithiolanyl) products $\mathbf{1 5}$ and $\mathbf{1 6}$ as white solids in 55\% and 73\% yield after purifications using column chromatography. The $\mathbf{1 6}$ structure is also confirmed by single crystal data. (Scheme 4).<smiles>COC(=O)c1c(N)cccc1NC(=O)C1SCCS1</smiles> 
In a similar procedure, compound $\mathbf{5}$ and $\mathbf{1 0}$ was reacted with 2.2 equivalents of $\alpha$ - thiophenyl acetyl chloride gives the corresponding esters $\mathbf{1 7}$ and $\mathbf{1 8}$ in 85\% yields each. The compound $\mathbf{1 8}$ structure is also confirmed by single crystal data. (Scheme 5).
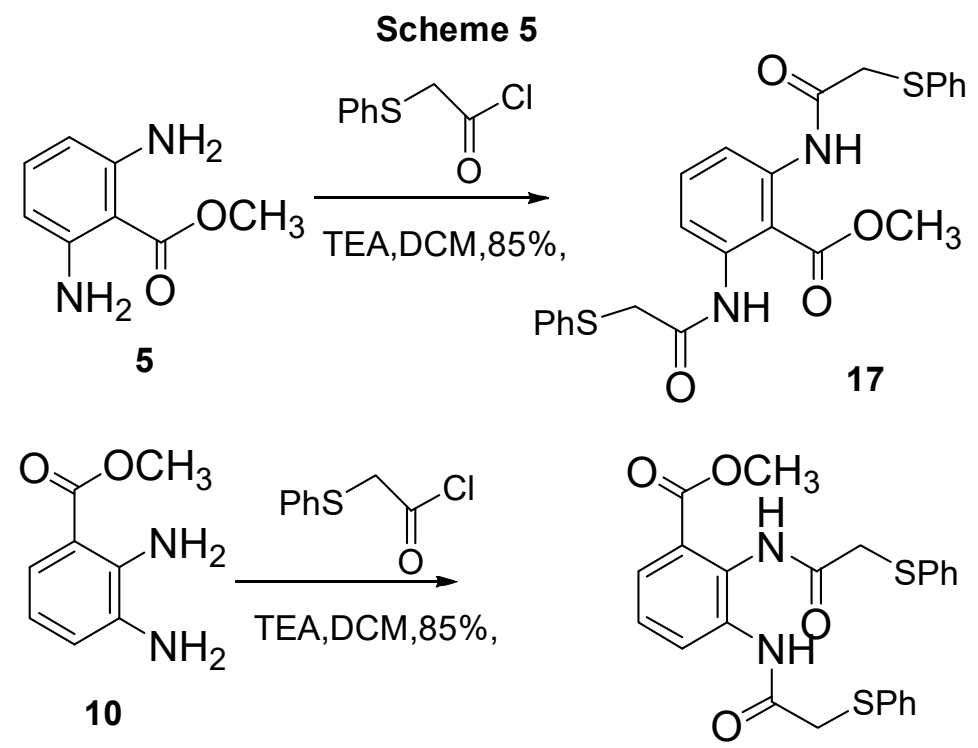

18

In a similar procedure, compound $\mathbf{5}$ was reacted with 2.2 equivalents of $\alpha$-benzyloximino acid chloride $^{22}$. After chromatographic separation, 2,6-bis-benzyloxy methylester 19 was isolated in $66 \%$ yield along with the 6-mono-benzyloxy methylester $\mathbf{2 0}$ in $5 \%$ yields. Both compounds were isolated as white solids. (Scheme 6).

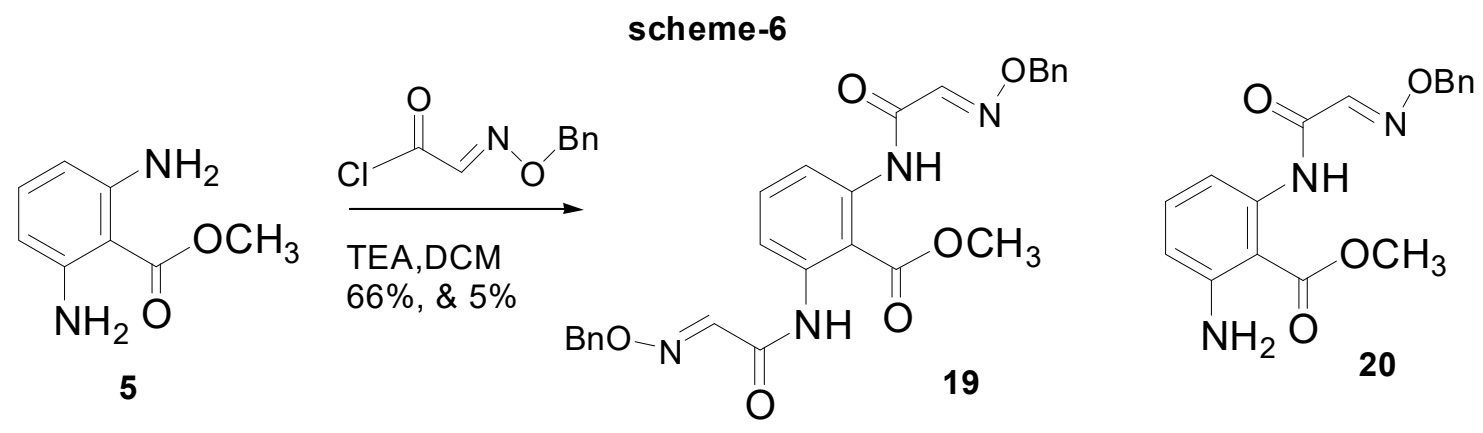

We also attempted to synthesize the 2,3-bis-benzyloxy methyl ester $\mathbf{2 2}$ from direct nucleophilic substitution reactions of diamine $\mathbf{1 0}$ with $\alpha$-benzyloximino acid chloride in either of TEA or 
pyridine and sodium hydride method. In both cases, our attempts were unsuccessful and resulted in either the isolation of starting materials or unidentified mixtures of products. But we were successful in synthesizing the $\mathbf{2 2}$ in two steps via acetonide protection (Scheme 7). Thus, reaction of $\mathbf{1 0}$ with 2,2-dimethoxy propane in presence catalytic amount of para toluene sulfonic acid gave $\mathbf{2 1}$ as residual oil in $47 \%$ yield after column chromatography purification, and reaction with $\alpha$-benzyloximino acid chloride ${ }^{22}$ and workup with dil $\mathrm{HCl}$ gave $\mathbf{2 2}$ as white solid in $78 \%$ yield after chromatographic purification (Scheme 7).

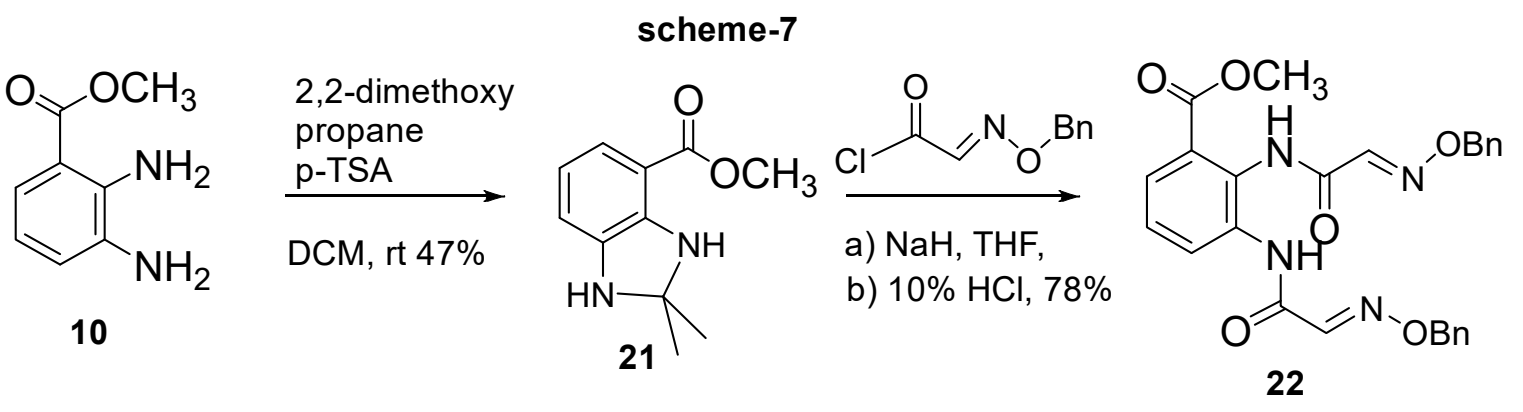

\section{Different substitution at the 2- and 6-positions}

As mentioned above, the synthesis of 2,6-bis-benzyloxy methylester 19 was accompanied by the isolation of the 6-mono-benzyloxy methyl ester 20. Recognizing that 6-mono-benzyloxy methyl ester 20 could lead to derivatives with a different substituent at the 6-position, we sought to increase the yield of 20. Accordingly, diamine $\mathbf{5}$ was reacted with 1.2 equivalents of $\alpha$ benzyloximino acid chloride ${ }^{22}$. Column chromatographic purification resulted in the isolation of the desired 6-mono benzyloxy methyl ester $\mathbf{2 0}$ in $62 \%$ yield along with bis-benzyloxy methylester 19 in $15 \%$ yield. Subsequent reaction of $\mathbf{2 0}$ with 1,3-dithiolane-2-carbonyl chloride ${ }^{17}$ led to new ditopic derivative $\mathbf{2 3}$ as pale-yellow solid in $64 \%$ yield after column chromatographic purification (scheme 8). 


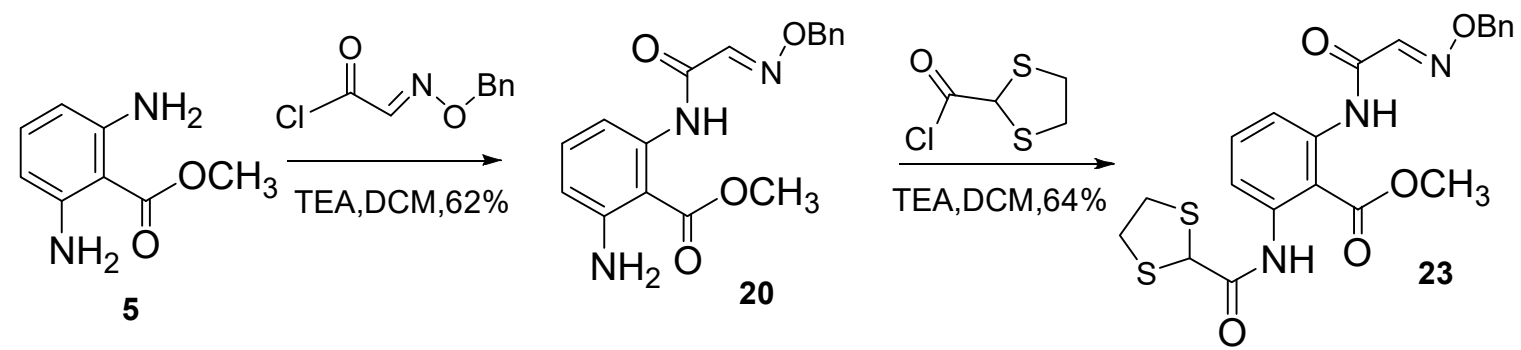

It is worth to mentioned that the while doing the Curtius rearrangement on compound $\mathbf{3}$, on heating at $110^{\circ} \mathrm{C}$ gives urea derivatives, which is common intermediate step for the synthesis of quinazoline derivatives which having biological properties ${ }^{23-24}$. Under acidic condition 24 gives benzo[d][1,3]oxazin-4-one $\mathrm{e}^{25}$ and in basic condition gives 2,4-quinazolindione derivatives ${ }^{23}$ respectively (scheme-9).

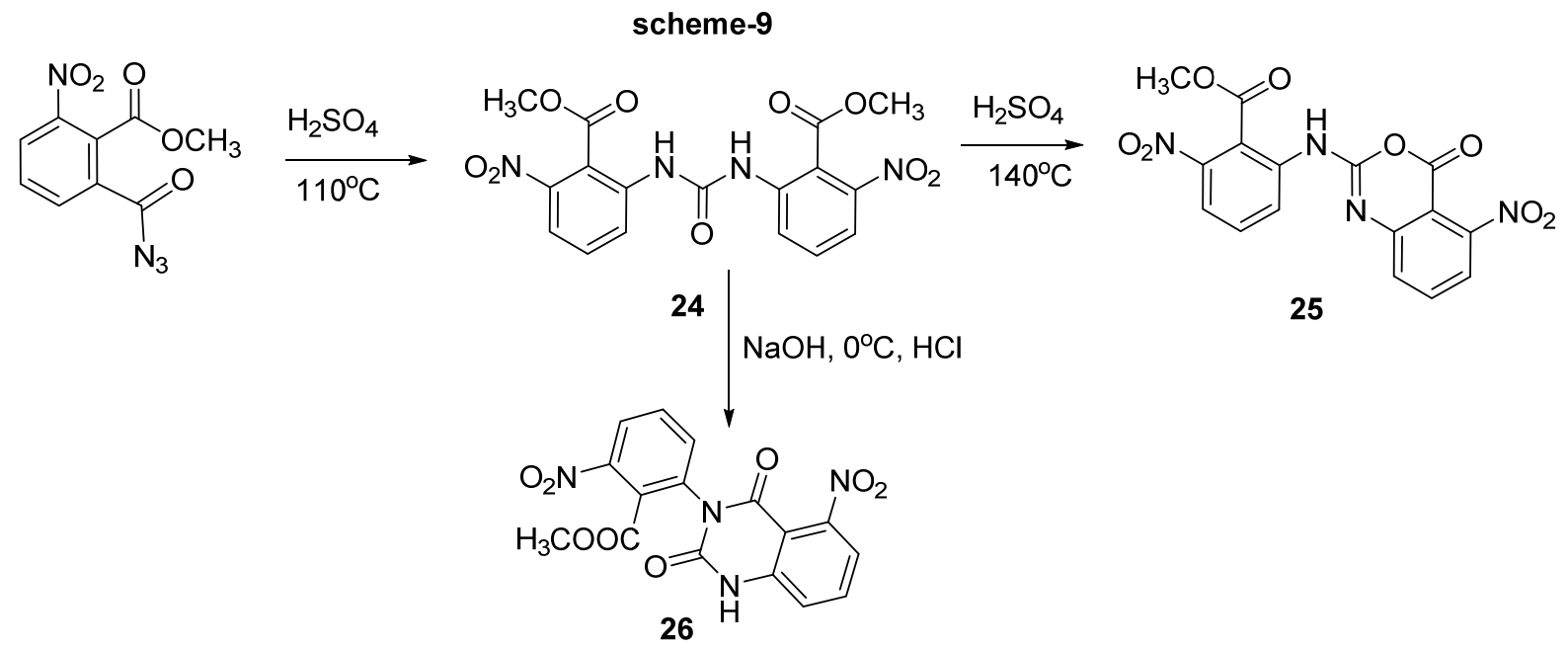

All new compounds were isolated as air- and moisture-stable solids. All compounds were fully characterized using ${ }^{1} \mathrm{H}$ and ${ }^{13} \mathrm{C}$ NMR spectroscopy and elemental analysis. The solubilities were excellent in common organic solvents, but $\mathbf{1 3}$ and $\mathbf{2 4}$ was soluble only in DMSO.

Preliminary experiments in which these compounds were mixed with various silver salts resulted in the formation of insoluble suspensions that could not be recrystallized for X-ray 
crystallographic analysis. However, elemental analysis of the isolated suspensions confirms the presence of silver, suggesting that complexation reactions are indeed occurring.

\section{OPTIC PROPERTIES}

TABLE: Uv-Vis Studies of compounds

\begin{tabular}{|l|l|l|l|l|l|l|l|}
\hline entry & compound & $\boldsymbol{\lambda}$ & $\boldsymbol{\varepsilon}$ & $\boldsymbol{\lambda}$ & $\boldsymbol{\varepsilon}$ & $\boldsymbol{\Lambda}$ & $\boldsymbol{\varepsilon}$ \\
\hline 1 & 5 & 206.52 & 20672.1875 & 249.95 & 12455.0434 & 348.52 & 5110.4080 \\
\hline 2 & 10 & 194.23 & 12641.0729 & 232.87 & 17762.0000 & 348.87 & 4370.4688 \\
\hline 3 & 13 & 192.31 & 25846.3190 & 236.29 & 16456.5333 & & \\
\hline 4 & 15 & 191.92 & 23265.5382 & 240.47 & 4003.2552 & 271.09 & 5111.8490 \\
\hline 5 & 16 & 193.08 & 20814.8352 & 227.92 & 22114.2857 & & \\
\hline 6 & 17 & 191.92 & 21302.8571 & 238.57 & 17375.1429 & & \\
\hline 7 & 18 & 193.08 & 6515.9828 & 225.25 & 3893.0573 & & \\
\hline 8 & 19 & 192.69 & 109777.916 & 208.81 & 76122.4965 & 332.28 & 21387.4167 \\
\hline 9 & 20 & 191.54 & 34272.9576 & 231.34 & 24875.3571 & 360.99 & 8007.1205 \\
\hline 10 & 21 & 191.54 & 21627.6855 & 227.54 & 19688.2910 & & \\
\hline 11 & 22 & 191.92 & 29271.6667 & 258.65 & 7661.3333 & & \\
\hline 12 & 23 & 191.92 & 65453.3073 & 211.88 & 33804.6484 & & \\
\hline
\end{tabular}

The optical properties of compounds are of primary concern in chromogenic sensing and patterning. The solution-phase UV-vis absorption spectrum was recorded at room temperature in dilute chloroform solutions of about $10^{-5} \mathrm{M}$ concentration. As shown in table, In the absorption spectra for all compounds shows around $192 \mathrm{~nm}$ band seems to be an inherent property of these compounds and is not due to impurities as was verified by thin layer chromatography and NMR 
spectroscopy. The absorption maximum of $\mathbf{5}$ and $\mathbf{1 0}$ was remarkably blue shift shows at $348 \mathrm{~nm}$ each. But 2,6, disubstituted derivatives 15 and $\mathbf{1 9}$ shows absorption maximum at 271 and $332 \mathrm{~nm}$ respectively, the decrease of absorption maximum to 270 in case of $\mathbf{1 5}$ can be explained that it contains dithialone moiety, is responsible for it. Similarly, the compound $\mathbf{2 0}$ with a free amine group exhibited max at $360 \mathrm{~nm}$, which is close to its parents compound $\mathbf{5}$ which having two free amines. Compound 19 compared with the absorption of corresponding its isomer compound 23 with the same chromophore showed a red-shifted absorption (no peaks and $443 \mathrm{~nm}$, respectively), presumably due to extension of ineffective p-conjugation in ortho isomers.

\section{Conclusions}

We have prepared a series of new compounds containing dithiolane or benzyloxy oximate to aromatic rings bonded to substituted anthranilic acid core. The synthetic procedure is straightforward, and the products are obtained in good to excellent yields after chromatographic purification. Excellent solubility properties and the presence of electron donor groups in the modified anthranilic acid derivative described herein may be advantageous in applications such as anthranilic acid derivatives-metal conjugate synthesis, for example. The efficiency of chromogenic sensing is more remarkably affected by the chemical environment of the anthranilic acid derivatives, depending on the presence of a protecting amino group or amide group. Taking into account these considerations, it is possible to optimize the uv-vis sensing of the molecules by structure modification. All these compounds possess multiple sulfur atoms and are thus capable of binding in a multidentate fashion to soft transition metal ions. A reaction of these ligands with late transition metal ions is a current focus in our laboratory. 


\section{ACKNOWLEDGMENTS}

The authors thank the National Science Foundation and the Welch Foundation for support of this work.

Declaration of Competing Interest The authors declare that they have no known competing financial interests

Data and materials availability: Requests for materials should be addressed to Sudershan Gondi (gondisr@gmail.com)

\section{EXPERIMENTAL SECTION:}

3-Nitrophthalic anhydride and 3-Nitrophthalic acid were obtained from Acros and Aldrich respectively. All other materials were reagent grade unless otherwise specified. All reactions were carried out in a dry nitrogen atmosphere. ${ }^{1} \mathrm{H}$ and ${ }^{13} \mathrm{C}$ NMR spectra were obtained on a 400MHz Bruker Avance NMR spectrometer. Infrared spectra were obtained on a Nicolet Magna-IR 560 spectrometer E.S.P. Elemental analyses were obtained with a CE Elantech ThermoFinnigan Flash 1112 CHN elemental analyzer. Melting points were collected on a TA Instruments DSC 2010 Differential Scanning Calorimeter using a heating rate of $108^{\circ} \mathrm{C} / \mathrm{min}$ and nitrogen as a purge gas. 2-Carbomethoxy-3-nitro-benzoic acid (1), ${ }^{26}$-carbomethoxy-3-nitrobenzoyl chloride (2), ${ }^{27}$ 2-azidocarbonyl-6-nitro-benzoic acid methyl ester (3), ${ }^{28}$ 2-amino-6-nitrobenzoic acid methyl ester (4), ${ }^{29}$ 2,6-diamino benzoic acid methyl ester (5), ${ }^{30}$ 2-Carbomethoxy-6nitro-benzoic acid (6), ${ }^{31}$ 2-carbomethoxy-6-nitro-benzoyl chloride(7), ${ }^{32}$ 2-azidocarbonyl-3-nitrobenzoic acid methyl ester(8), ${ }^{33}$ 2-amino-3-nitrobenzoic acid methyl ester (9), ${ }^{30}$ 2,3-diamino 
benzoic acid methyl ester $(10),{ }^{34}$ were prepared with modified methods and spectral data is presented. $\alpha$-Benzyloximino acid chloride ${ }^{22}$ and 1,3-dithiolane-2-carbonyl chloride ${ }^{16}$ were prepared as described previously.

2-Carbomethoxy-3-nitro-benzoic acid $^{26}$ (1): This procedure is modified from an earlier<smiles>COC(=O)c1c(C(=O)O)cccc1[N+](=O)[O-]</smiles>
reported procedure. ${ }^{26}$ 3- nitro-phthalic anhydride $(30 \mathrm{~g}, 155 \mathrm{mmol})$ was refluxed in absolute methanol $(320 \mathrm{~mL})$ for $24 \mathrm{~h}$. Thereafter methanol was distilled out to get the mixture of products 2 and $\mathbf{6}$ in 9.5:0.5 ratio $(35.0 \mathrm{~g}, 99$ $\%$ ) as white solid, used in next reaction without purification. Mp $144.74^{\circ} \mathrm{C}$. (lit

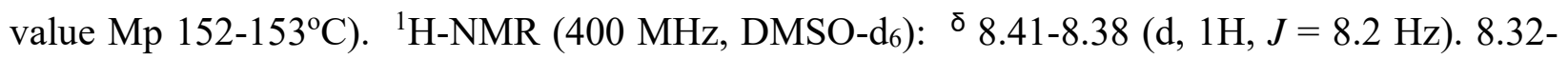
$8.30(\mathrm{~d}, 1 \mathrm{H}, J=7.8 \mathrm{~Hz}), 7.87-7.83(\mathrm{t}, 1 \mathrm{H}, J=8.0 \mathrm{~Hz}), 3.87\left(\mathrm{~s}, 3 \mathrm{H}, \mathrm{COOCH}_{3}\right) .{ }^{13} \mathrm{C}-\mathrm{NMR}(100.6$ MHz, DMSO-d $)^{\circ}{ }^{\delta} 166.2,166.0,146.9,136.5,132.1,132.0,130.2,129.0,53.9$. IR (KBr): 3097, $2957,1736,1572,1536,1477,1279 \mathrm{~cm}^{-1}$.

2-Carbomethoxy-3-nitro-benzoyl chloride ${ }^{27}$ (2): This procedure is modified from an earlier<smiles>COC(=O)c1c(C(=O)Cl)cccc1[N+](=O)[O-]</smiles>
reported procedure ${ }^{27}$. The reaction mixture 1 and $6(30 \mathrm{~g}, 133 \mathrm{mmol})$ and thionyl chloride $(29.2 \mathrm{~mL}, 47.6 \mathrm{~g}, 399 \mathrm{mmol})$ were heated at reflux for two hours, and the excess thionyl chloride removed by distillation under reduced pressure on rota evaporator to obtained the yellow residue. The residue was evaporated in vacuum three times with $50 \mathrm{~mL}$ of dry toluene to remove all traces of thionyl chloride. The residue solidified upon cooling. Recrystallization from toluene gives $15.5 \mathrm{~g}$ of white crystals products 2 and 7 in (9.5:0.5 ratio) in 95\% yield. Mp 72-74 ${ }^{\circ} \mathrm{C}$. (lit value Mp 7879.5 $\left.{ }^{\circ} \mathrm{C}\right) .{ }^{1} \mathrm{H}-\mathrm{NMR}\left(400 \mathrm{MHz}, \mathrm{CDCl}_{3}\right):{ }^{\delta} 8.55-8.53(\mathrm{~d}, 1 \mathrm{H}, J=7.9 \mathrm{~Hz}), 8.45-8.43(\mathrm{~d}, 1 \mathrm{H}, J=8.2$ $\mathrm{Hz}), 7.86-7.81(\mathrm{t}, 1 \mathrm{H}, J=8.2 \mathrm{~Hz}), 3.98\left(\mathrm{~s}, 3 \mathrm{H}, \mathrm{COOCH}_{3}\right) .{ }^{13} \mathrm{C}-\mathrm{NMR}\left(100.6 \mathrm{MHz}, \mathrm{CDCl}_{3}\right):{ }^{\delta}$ 
166.2, 165.0, 145.6, 138.0, 133.6, 131.4, 130.8, 130.4, 54.2. IR (KBr): 3097, 2957, 1736, 1572, $1540,1472,1284 \mathrm{~cm}^{-1}$.

2-Azidocarbonyl-6-nitro-benzoic acid methyl $\operatorname{ester}^{28}$ (3): This procedure is modified from an<smiles>COC(=O)c1c(C(N)=O)cccc1[N+](=O)[O-]</smiles>
earlier reported procedure. ${ }^{28}$ To a solution of reaction mixture 2 and 7 (30 g, $123 \mathrm{mmol})$ in $150 \mathrm{~mL}$ of dry acetone, pre-cooled to $0^{\circ} \mathrm{C}$, was added $(32.1 \mathrm{~g}$, $494 \mathrm{mmol}$ ) of sodium azide. After 15 minutes of stirring, the reaction mixture was diluted by addition of water $(100 \mathrm{~mL})$ and stirred at same temperature for $1 \mathrm{~h}$, during this time product solidified. Filter at pump, washed with water $(100 \mathrm{~mL})$ and hexane $(100 \mathrm{~mL})$ to obtained mixture of product 3 and $\mathbf{8}$ (in 9.5:0.5 ratio) as yellow solid. (29.3 g, 97\%). $\mathrm{Mp} 80-82^{\circ} \mathrm{C}$ which is used in next reaction without purification. ${ }^{1} \mathrm{H}-\mathrm{NMR}\left(400 \mathrm{MHz}, \mathrm{CDCl}_{3}\right):{ }^{\delta}$ 8.41-8.39 (d, 1H, $J=8.2 \mathrm{~Hz}), 8.31-8.29(\mathrm{~d}, 1 \mathrm{H}, J=7.8 \mathrm{~Hz}), 7.73-7.69$ (t, $1 \mathrm{H}, J=8.0 \mathrm{~Hz}), 4.02$ (s, 3H, $\left.\mathrm{COOCH}_{3}\right) .{ }^{13} \mathrm{C}-\mathrm{NMR}\left(100.6 \mathrm{MHz} \mathrm{CDCl}_{3}\right):{ }^{\delta} 169.9,165.3,146.4,135.3,130.8,130.3$, 130.0, 129.2, 53.5. IR (KBr): 3102, 2955, 2158, 1755, 1693, 1539, 1354, $1259 \mathrm{~cm}^{-1}$.

2-Amino-6-nitro-benzoic acid methyl $\operatorname{ester}^{29}$ (4): This procedure is modified from an earlier

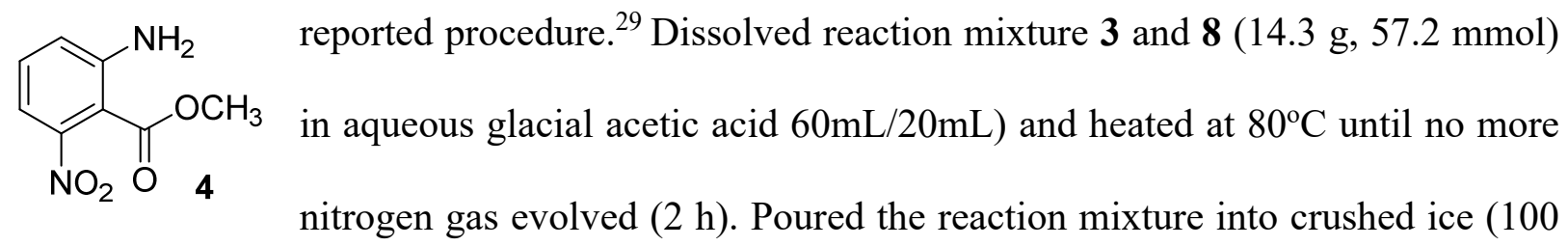
g) and stirred for 30 minutes to precipitate the product. Filtered and washed well with water (250 $\mathrm{mL})$. The solid residue was taken up in ethyl acetate $(100 \mathrm{~mL})$, washed with water $(100 \mathrm{~mL})$, brine solution $(100 \mathrm{~mL})$, dried over anhydrous $\mathrm{MgSO}_{4}$ and filter. All volatiles were removed, and the residue was purified using column chromatography (70:30 EtOAc:hex) to give 4 as yellow solid (7.85 g, 70\%). Mp 109.13ㄷ. (lit value Mp 108-110 $\left.{ }^{\circ} \mathrm{C}\right) .{ }^{1} \mathrm{H}-\mathrm{NMR}(400 \mathrm{MHz}$, $\left.\mathrm{CDCl}_{3}\right):{ }^{\delta} 7.19-7.15(\mathrm{t}, 1 \mathrm{H}, J=7.9 \mathrm{~Hz}), 6.92-6.90(\mathrm{~d}, 1 \mathrm{H}, J=7.8 \mathrm{~Hz}), 6.80-6.78(\mathrm{~d}, 1 \mathrm{H}, J=8.4$ 
$\mathrm{Hz}), 5.26$ (br-s, 2H, NH 2$), 3.73\left(\mathrm{~s}, 3 \mathrm{H}, \mathrm{COOCH}_{3}\right) .{ }^{13} \mathrm{C}-\mathrm{NMR}\left(100.6 \mathrm{MHz}, \mathrm{CDCl}_{3}\right):{ }^{\delta} 165.9$ 151.4, 148.8, 131.8, 120.4, 112.2, 106.8, 52.5. IR (KBr): 3492, 3385, 3103, 2955, 1716, 1624 1514, 1344, 1285, $836 \mathrm{~cm}^{-1}$. Elemental Analysis: Calcd for $\mathrm{C}_{8} \mathrm{H}_{8} \mathrm{~N}_{2} \mathrm{O}_{4}:$ C, 48.98; H, 4.11, N, 14.28. Found: C, 49.32; H, 4.01; N, 14.29. And eluted with (20:80 EtOAc:hex) to give product 9 as yellow solid $(0.2 \mathrm{~g}, 5 \%)$. Mp 97.28 $\mathrm{C}$. (lit value $\mathrm{Mp} 95-97^{\circ} \mathrm{C}$ ).

2, 6-Diamino benzoic acid methyl $\operatorname{ester}^{30}$ (5): This procedure is modified from an earlier reported procedure. ${ }^{30}$ To a solution of 2-amino-6-nitro-benzoic acid methyl<smiles>COC(=O)c1c(N)cccc1N</smiles>
ester (4) $(1.0 \mathrm{~g}, 5.1 \mathrm{mmol})$ in $80 \mathrm{~mL}$ of methanol, $50 \mathrm{mg}$ of $10 \%$ palladium carbon was added. The reaction mixture stirred under hydrogen atmosphere until the starting material disappeared $(18 \mathrm{~h})$. After completion of reaction, filter through celite pad and wash with additional methanol $(50 \mathrm{~mL})$. The filtrate on concentration, the residue was purified by column chromatography (40:60 EtOAc:hex) to give 5 as brown solid $(0.76 \mathrm{~g}, 90 \%)$. Mp $81.61^{\circ} \mathrm{C}$. (lit value $\mathrm{Mp} 80-82^{\circ} \mathrm{C}$ ). ${ }^{1} \mathrm{H}-\mathrm{NMR}(400 \mathrm{MHz}$, $\left.\mathrm{CDCl}_{3}\right):{ }^{\delta} 6.98-6.94(\mathrm{t}, 1 \mathrm{H}, J=8.0 \mathrm{~Hz}), 5.97-5.95(\mathrm{~d}, 2 \mathrm{H}, J=8.0 \mathrm{~Hz}), 3.91\left(\mathrm{~s}, 3 \mathrm{H}, \mathrm{COOCH}_{3}\right)$. ${ }^{13} \mathrm{C}-\mathrm{NMR}\left(100.6 \mathrm{MHz}, \mathrm{CDCl}_{3}\right):{ }^{\delta}$ 169.1, 151.2, 133.9, 104.6, 97.6, 51.2. IR (KBr): 3481, 3364, 2946, 1664, 1576, 1458, 1347, $805 \mathrm{~cm}^{-1}$. Elemental Analysis: Calcd for $\mathrm{C}_{8} \mathrm{H}_{10} \mathrm{~N}_{2} \mathrm{O}_{2}$ : C, 57.82; H, 6.07; N, 16.86. Found: C, 58.19; H, 6.18; N, 16.72 .

2-Carbomethoxy-6-nitro-benzoic acid $^{31}$ (6): This procedure is modified from an earlier reported procedure. ${ }^{31}$ 3-nitro-phthalic acid $(3.4 \mathrm{~g}, 21.3 \mathrm{mmol})$ was refluxed in
$\mathbf{6}$ $1 \mathrm{H}, J=7.2 \mathrm{~Hz}), 7.80-7.78(\mathrm{~d}, 1 \mathrm{H}, J=7.7 \mathrm{~Hz}), 3.82\left(\mathrm{~s}, 3 \mathrm{H}, \mathrm{COOCH}_{3}\right) .{ }^{13} \mathrm{C}-\mathrm{NMR}(100.6 \mathrm{MHz}$, 
DMSO-d6): ${ }^{\delta} 166.5,165.5,147.3,135.6,131.6,131.3,130.7,128.8,53.8$. IR (KBr): 3089, 2960, $2664,1732,1532,1350,1278 \mathrm{~cm}^{-1}$.

2-Carbomethoxy-6-nitro-benzoyl chloride ${ }^{32}$ (7): This procedure is modified from an earlier<smiles>COC(=O)c1cccc([N+](=O)[O-])c1C(=O)Cl</smiles>
reported procedure. ${ }^{32}$ In the same manner described for $\mathbf{2}$ and $\mathbf{6}, 2$ carbomethox-3-nitro benzoic acid (6) $(4.5 \mathrm{~g}, 20.0 \mathrm{mmol})$ and thionyl chloride (4.4 $\mathrm{mL}, 7.13 \mathrm{~g}, 60.0 \mathrm{mmol}$ ) to gives 7 as yellow crystals. (4.60 g, 96\%). Mp 93-95 ${ }^{\circ}$ C. (lit value $\left.\mathrm{Mp} 97-99^{\circ} \mathrm{C}\right) .{ }^{1} \mathrm{H}-\mathrm{NMR}\left(400 \mathrm{MHz}, \mathrm{CDCl}_{3}\right){ }^{\circ}$ 8.40-8.38 (d, $1 \mathrm{H}, J=8.4 \mathrm{~Hz}), 8.39-8.35(\mathrm{~d}, 1 \mathrm{H}, J=8.6 \mathrm{~Hz}), 7.75-7.71(\mathrm{t}, 1 \mathrm{H}, J=8.0 \mathrm{~Hz}), 3.93(\mathrm{~s}, 3 \mathrm{H}$, $\left.\mathrm{COOCH}_{3}\right) .{ }^{13} \mathrm{C}-\mathrm{NMR}\left(100.6 \mathrm{MHz}, \mathrm{CDCl}_{3}\right):{ }^{\delta}$ 164.6, 163.3, 144.2, 136.2, 133.6, 131.3, 128.7 , 128.3, 53.4. IR (KBr): 3089, 2961, 980, 754, 1803, 1728, 1545, 1344, $1287 \mathrm{~cm}^{-1}$. Elemental Analysis: Calcd: C, 44.37; H, 2.48; N, 5.75. Found: C, 44.89; H, 2.46; N, 5.79.

2-Azidocarbonyl-3-nitro-benzoic acid methyl $\operatorname{ester}^{33}(8)$ : To a solution of 2-carbomethoxy-6-<smiles>COC(=O)c1cccc([N+](=O)[O-])c1C(N)=O</smilesnitro-benzoyl chloride (7) $(4.5 \mathrm{~g}, 18.0 \mathrm{mmol})$ in $150 \mathrm{~mL}$ of dry acetone, precooled to $0^{\circ} \mathrm{C}$, was added $(4.81 \mathrm{~g}, 70.0 \mathrm{mmol})$ of sodium azide. After 15 minutes of stirring, the reaction mixture was diluted by addition of water and stirred at same temperature for $1 \mathrm{~h}$ during this time product solidified. Filtered at pump, dissolve the residue solid in dichloromethane $(100 \mathrm{~mL})$. The organic layer was washed with water $(100 \mathrm{~mL})$, saturated sodium bicarbonate solution $(100 \mathrm{~mL})$, brine solution $(100 \mathrm{~mL})$ and dried over $\mathrm{MgSO}_{4}$. After filtration, evaporation and purification through column chromatography (20:80 EtOAc:hex) gave 8 as yellow solid $(4.30 \mathrm{~g}, 94 \%) . \mathrm{Mp} 88-90^{\circ} \mathrm{C} .{ }^{1} \mathrm{H}-$ NMR (400 MHz, $\left.\mathrm{CDCl}_{3}\right){ }^{\delta} 8.31-8.29(\mathrm{~d}, 1 \mathrm{H}, J=8.2 \mathrm{~Hz}), 8.17-8.15(\mathrm{~d}, 1 \mathrm{H}, J=6.9 \mathrm{~Hz}), 6.59-$ $6.55(\mathrm{t}, 1 \mathrm{H}, J=8.0 \mathrm{~Hz}), 3.84\left(\mathrm{~s}, 3 \mathrm{H}, \mathrm{COOCH}_{3}\right) .{ }^{13} \mathrm{C}-\mathrm{NMR}\left(100.6 \mathrm{MHz}, \mathrm{CDCl}_{3}\right):{ }^{\delta}$ 167.3, 147.1, 
139.2, 133.1, 132.1, 114.3, 113.8, 52.2. IR (KBr): 3085, 2956, 2271, 2176, 3451, 3332, 1728, $1701,1618,1570,1241,1110 \mathrm{~cm}^{-1}$.

2-Amino-3-nitro-benzoic acid methyl $\operatorname{ester}^{29}$ (9): In the same manner described for 2-amino-6-<smiles>COC(=O)c1cccc([N+](=O)[O-])c1N</smilesnitro-benzoic acid methyl ester (4), 2-azidocarbonyl-3-nitro-benzoic acid methyl ester (8) (4.0 g, $16.0 \mathrm{mmol})$ was treated aqueous glacial acetic acid. Column chromatographic purification (20:80 EtOAc:hex) gave 9 as yellow solid. (2.8 g, 89\%). Mp 94-96º C. (lit value Mp 95-97 C). ${ }^{1} \mathrm{H}-\mathrm{NMR}(400 \mathrm{MHz}$, $\left.\mathrm{CDCl}_{3}\right):{ }^{\delta} 8.30-8.28(\mathrm{~d}, 1 \mathrm{H}, J=8.4 \mathrm{~Hz}), 8.16-8.14(\mathrm{~d}, 1 \mathrm{H}, J=7.7 \mathrm{~Hz}), 6.59-6.55(\mathrm{t}, 1 \mathrm{H}, J=8.2$ $\mathrm{Hz}), 3.84\left(\mathrm{~s}, 3 \mathrm{H}, \mathrm{COOCH}_{3}\right) .{ }^{13} \mathrm{C}-\mathrm{NMR}\left(100.6 \mathrm{MHz}, \mathrm{CDCl}_{3}\right):{ }^{\delta} 167.4,147.2,139.3,133.2$, 132.2, 114.4, 113.9, 52.2. IR (KBr): 3453, 3318, 3102, 2964, 1701, 1619, 1515, 1254, $883 \mathrm{~cm}^{-1}$. Elemental Analysis: Calcd for $\mathrm{C}_{8} \mathrm{H}_{8} \mathrm{~N}_{2} \mathrm{O}_{4}$ : C, 48.98; H, 4.11; N, 14.28. Found: C, 49.09; H, $4.02 ; \mathrm{N}, 14.22$.

2, 3-Diamino benzoic acid methyl $\operatorname{ester}^{34}$ (10): In the same manner described for 2, 6-diamino<smiles>COC(=O)c1cccc(N)c1N</smiles>
benzoic acid methyl ester (5), the 2-amino-3-nitro-benzoic acid methyl ester (9) $(2.0 \mathrm{~g}, 10.0 \mathrm{mmol})$ was treated with $100 \mathrm{mg}$ of $10 \%$ palladium over charcoal in $80 \mathrm{~mL}$ of methanol. Column chromatographic purification (30:70 EtOAc:hex) gave 10 as brown solid. (1.55 g, 91\%). Mp 64.24 ${ }^{\circ} \mathrm{C}$. (lit value Mp 63-64 $\left.{ }^{\circ} \mathrm{C}\right) .{ }^{1} \mathrm{H}-\mathrm{NMR}(400$ $\left.\mathrm{MHz}_{\mathrm{CDCl}}\right) \mathrm{C}^{\delta} 7.49-7.47(\mathrm{~d}, 1 \mathrm{H}, J=8.1 \mathrm{~Hz}), 6.83-6.81(\mathrm{~d}, 1 \mathrm{H}, J=7.5 \mathrm{~Hz}), 6.61-6.57(\mathrm{t}, 1 \mathrm{H}, J$ $=7.7 \mathrm{~Hz}), 4.43\left(\mathrm{br}-\mathrm{s}, 4 \mathrm{H}, \mathrm{NH}_{2}\right), 3.91\left(\mathrm{~s}, 3 \mathrm{H}, \mathrm{COOCH}_{3}\right) .{ }^{13} \mathrm{C}-\mathrm{NMR}\left(100.6 \mathrm{MHz}, \mathrm{CDCl}_{3}\right):{ }^{\delta} 168.8$, 140.9, 134.1, 122.3, 120.4, 116.5, 111.6, 51.4. IR (KBr): 3428, 3368, 1701, 1693, 1560, 1473, 1287, 2947, $854 \mathrm{~cm}^{-1}$. Elemental Analysis: Calcd for $\mathrm{C}_{8} \mathrm{H}_{10} \mathrm{~N}_{2} \mathrm{O}_{2}:$ C, 57.82; H, 6.07; N, 16.86 . Found: C, 57.85; H, 6.01; N, 16.75. and eluted with (20:80 EtOAc:hex) to give corresponding acetonide 16 as residual oil (70 $\mathrm{mg}, 5 \%)$. 
2,6-Bis-(2-bromo-acetylamino)-benzoic acid methyl ester (11): Dissolved 2,6-bis-aminobenzoic acid methyl ester (5) (1.0 g, $6.0 \mathrm{mmol})$ in dichloromethane (40<smiles>COC(=O)c1c(NC(=O)CBr)cccc1NC(=O)CBr</smiles>
$\mathrm{mL})$ and cooled in a water/ice bath. Triethylamine $(1.85 \mathrm{~mL}, 1.33 \mathrm{~g}$, $13.2 \mathrm{mmol}$ ) was then added, and the resulting suspension was stirred for $15 \mathrm{~min}$. A solution of bromo-acetyl bromide $(2.67 \mathrm{~g}, 13.2 \mathrm{~mol})$ in dichloromethane $(15 \mathrm{~mL})$ was added dropwise over a period of $15 \mathrm{~min}$, and the resulting mixture was allowed to warm to room temperature and stirred for $6 \mathrm{~h}$. The reaction mixture was quenched by the addition of aqueous solution of $\mathrm{HCl}(50 \mathrm{~mL})$. The aqueous solution was extracted with dichloromethane $(100 \mathrm{~mL})$, and the organic layer was washed with water $(100 \mathrm{~mL})$, saturated sodium bicarbonate solution $(100 \mathrm{~mL})$, water again $(100$ $\mathrm{mL})$, and brine $(100 \mathrm{~mL})$ dried over anhydrous $\mathrm{MgSO}_{4}$ and filtered. Removal of volatiles and the residue was purified using column chromatography ( $\mathrm{SiO}_{2}, 15: 85$ EtOAc:hex) to give $\mathbf{1 1}$ as white solid. (1.70 g, 69\%). Mp 150.59 ${ }^{\circ} \mathrm{C} .{ }^{1} \mathrm{H}-\mathrm{NMR}\left(400 \mathrm{MHz}, \mathrm{CDCl}_{3}\right):{ }^{\delta} 10.6$ (br-s, $\left.2 \mathrm{H}, \mathrm{NH}\right)$, 8.20-8.18 (d, 2H, $J=8.4 \mathrm{~Hz}), 7.56-7.52(\mathrm{t}, 1 \mathrm{H}, J=8.4 \mathrm{~Hz}), 4.11\left(\mathrm{~s}, 3 \mathrm{H}, \mathrm{COOCH}_{3}\right), 4.06(\mathrm{~s}, 4 \mathrm{H}$, $\left.\mathrm{CH}_{2} \mathrm{Br}\right) .{ }^{13} \mathrm{C}-\mathrm{NMR}\left(100.6 \mathrm{MHz}, \mathrm{CDCl}_{3}\right):{ }^{\delta} 166.9,164.1,138.6,133.7,118.0,108.8,53.4,29.7$. IR (KBr): 3309, 3263, 3036, 2960, 1713, 1676, 1525, 1467, $1260 \mathrm{~cm}^{-1}$. Elemental Analysis: Calcd for $\mathrm{C}_{12} \mathrm{H}_{12} \mathrm{Br}_{2} \mathrm{~N}_{2} \mathrm{O}_{4}$ : C, 35.32; H, 2.96; N, 6.87. Found: C, 35.17; H, 3.01; N, 6.68.

2,3-Bis[(-Bromo-acetyl)-amino]-benzoic acid methyl ester (12): In the manner described<smiles>COC(=O)c1cccc(NC(=O)CBr)c1NC(=O)CBr</smiles>
above, 2,3-bis-amino-benzoic acid methyl ester (10) (1.0 g, $6.0 \mathrm{mmol})$ was treated with bromo-acetyl bromide $(2.67 \mathrm{~g}, 13.2 \mathrm{mmol})$ in presence of $1.85 \mathrm{ml},(1.33 \mathrm{~g}, 13.2 \mathrm{mmol})$ of triethylamine at $0^{\circ} \mathrm{C}$ in $40 \mathrm{~mL}$ of dichloromethane. Column chromatography (20:80 EtOAc:hex) to give 12 as white solid (1.6 g, 65\%). Mp $164.18^{\circ} \mathrm{C} .{ }^{1} \mathrm{H}-\mathrm{NMR}$ (400 MHz, $\left.\mathrm{CDCl}_{3}\right)$ : ${ }^{\delta} 10.72$ (br-s, 1H, 
NH), 9.09 (br-s, 1H, NH), 7.95-7.93 (d, 2H, J = 7.8 Hz), 7.42-7.38 (t, 1H, $J=8.0 \mathrm{~Hz}), 4.12(\mathrm{~s}$, $\left.2 \mathrm{H}, \mathrm{CH}_{2} \mathrm{Br}\right), 4.01$ (s, $\left.2 \mathrm{H}, \mathrm{CH}_{2} \mathrm{Br}\right), 3.97\left(\mathrm{~s}, 3 \mathrm{H}, \mathrm{COOCH}_{3}\right) .{ }^{13} \mathrm{C}-\mathrm{NMR}\left(100.6 \mathrm{MHz}, \mathrm{CDCl}_{3}\right):{ }^{\delta}$ $167.7,166.5,165.2,132.3,132.0,131.5,129.1,126.7,123.0,53.2,29.6,28.9 . \mathrm{IR}(\mathrm{KBr}): 3448$, $3269,3008,2952,1720,1666,1546,1295$. Elemental Analysis: Calcd for $\mathrm{C}_{12} \mathrm{H}_{12} \mathrm{Br}_{2} \mathrm{~N}_{2} \mathrm{O}_{4}$ : C, 35.32; H, 2.96; N, 6.87. Found: C, 35.80; H, 3.14; N, 6.96.

2,6-Bis-[2-(pyridin-2-ylmethoxy)-acetylamino]-benzoic acid methyl ester (13): Pyridine

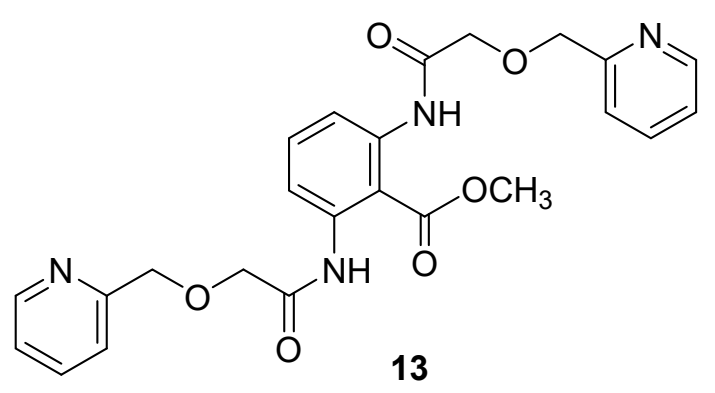
methylalcohol (588 mg, $5.4 \mathrm{mmol})$ was dissolved in THF $(10 \mathrm{~mL})$ and cooled in an ice bath. Sodium hydride (130 $\mathrm{mg}, 5.4 \mathrm{mmol})$ was added, and the mixture was stirred 15 minutes. $11(1.0 \mathrm{~g}, 2.4 \mathrm{mmol})$ was added portion wise over a period of 15 minutes.

The reaction mixture was stirred at room temperature for $24 \mathrm{~h}$. The THF was removed using a rotary evaporator and the residue was quenched by the addition of water $(100 \mathrm{~mL})$. The aqueous solution was extracted with ethyl acetate $(100 \mathrm{~mL})$, and the organic layer was washed with water $(100 \mathrm{~mL})$, saturated sodium bicarbonate solution $(100 \mathrm{~mL})$, water $(100 \mathrm{~mL})$, and brine $(100 \mathrm{~mL})$, and then dried over anhydrous $\mathrm{MgSO}_{4}$. After filtration and concentration of the filtrate, the residue was purified by column chromatography (20:80 EtOAc:hex) to give $\mathbf{1 3}$ as residual oil (0.67 g, 59\%). ${ }^{1} \mathrm{H}-\mathrm{NMR}\left(400 \mathrm{MHz}, \mathrm{CDCl}_{3}\right):{ }^{\delta} 10.7$ (br-s, $\left.2 \mathrm{H}, \mathrm{NH}\right), 8.53-8.52(\mathrm{~d}, 2 \mathrm{H}, J=3.4$ Hz), 8.20-8.18 (d, 2H, $J=8.3 \mathrm{~Hz}), 7.69-7.65$ (t, 1H, $J=7.6 \mathrm{~Hz}), 7.48-7.41$ (m, 3H), 7.19-7.17 (t, $2 \mathrm{H}, J=2.7 \mathrm{~Hz}), 4.73(\mathrm{~s}, 4 \mathrm{H}), 4.16(\mathrm{~s}, 4 \mathrm{H}), 3.61\left(\mathrm{~s}, 3 \mathrm{H}, \mathrm{COOCH}_{3}\right) .{ }^{13} \mathrm{C}-\mathrm{NMR}(100.6 \mathrm{MHz}$, $\left.\mathrm{CDCl}_{3}\right):^{\delta} 167.9,167.0,156.7,149.4,138.6,136.8,133.7,122.9,121.8,117.5,108.9,74.5,70.7$ 52.3. IR (KBr): $3361,2967,1694,1585,1469 \mathrm{~cm}^{-1}$ 


\section{2,6-Bis-[([1,3]dithiolane-2-carbonyl)-amino]-benzoic acid methyl ester (15):}

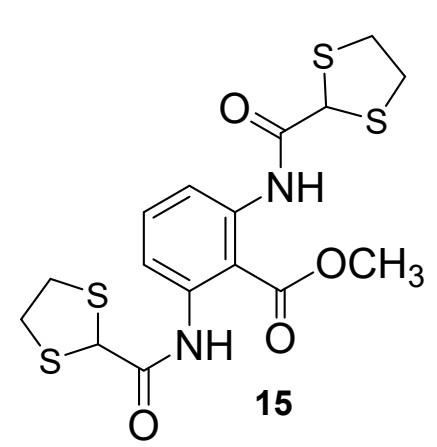

2,6-Bis-amino-benzoic acid methyl ester (5) $(0.5 \mathrm{~g}, 3.0 \mathrm{mmol})$ was dissolved in dichloromethane $(20 \mathrm{~mL})$ and cooled in an ice bath. Triethylamine $(1.67 \mathrm{~mL}, 1.21 \mathrm{~g}, 12.0 \mathrm{mmol})$ was added and the solution was stirred for 15 minutes. 1,3-dithiolane-2-carbonyl chloride $^{16}$ (1.22 g, $\left.7.2 \mathrm{mmol}\right)$ was dissolved in dichloromethane (5 $\mathrm{mL}$ ) and the solution was added dropwise over a period of 15 minutes. The reaction mixture was stirred in the ice bath for $1 \mathrm{~h}$ and stirred at room temperature overnight, and reaction mixture was quenched with aqueous solution of $\mathrm{HCl}$ and extracted with dichloromethane (50 $\mathrm{mL} \mathrm{X} \mathrm{2),} \mathrm{and}$ the organic layer was washed with water $(100 \mathrm{~mL})$, saturated sodium bicarbonate solution (100 $\mathrm{mL})$, water $(100 \mathrm{~mL})$, brine $(100 \mathrm{~mL})$, dried over anhydrous $\mathrm{MgSO}_{4}$ and filtered. All volatiles were removed and the residue was purified using column chromatography (20:80 EtOAc:hex) to give 15 as white solid $(0.4 \mathrm{~g}, 55 \%)$. Mp 152-154 ${ }^{\circ} \mathrm{C} .{ }^{1} \mathrm{H}-\mathrm{NMR}\left(400 \mathrm{MHz}, \mathrm{CDCl}_{3}\right):{ }^{\delta} 10.74$ (s, 2H, NH), 8.14-8.12 (d, 2H, $J=8.3 \mathrm{~Hz}), 7.52-7.48(\mathrm{t}, 1 \mathrm{H}, J=8.3 \mathrm{~Hz}), 5.01(\mathrm{~s}, 2 \mathrm{H}), 4.05(\mathrm{~s}, 3 \mathrm{H})$, 3.44-3.35 (m, 8H). ${ }^{13} \mathrm{C}-\mathrm{NMR}\left(100.6 \mathrm{MHz}, \mathrm{CDCl}_{3}\right):{ }^{\delta} 168.5,167.1,138.7,133.6,118.0,109.4$, 54.7, 53.1, 39.2. IR (KBr): 3292, 1695, 1678, 1584, 1466, 1273, 1074, $805 \mathrm{~cm}^{-1}$. Elemental Analysis: Calcd for $\mathrm{C}_{16} \mathrm{H}_{18} \mathrm{~N}_{2} \mathrm{O}_{4} \mathrm{~S}_{4}$ : C, 44.63; H, 4.21; N, 6.51. Found: C, 44.91; H, 3.92; N, 6.47 .

2,3-Bis-[([1,3]dithiolane-2-carbonyl)-amino]-benzoic acid methyl ester (16): In the manner<smiles>COC(=O)c1ccccc1NC(=O)C1SCCS1</smiles>
described above, 2,3-bis-amino-benzoic acid methyl ester (10) $(0.5 \mathrm{~g}, 3.0$ mmol) was treated with 1,3 -dithiolane-2-carbonyl chloride ${ }^{16}$ (1.22 g, 7.2 $\mathrm{mmol})$ in presence of $1.67 \mathrm{~mL},(1.21 \mathrm{~g}, 12 \mathrm{mmol})$ of triethylamine at $0^{\circ} \mathrm{C}$ in $20 \mathrm{~mL}$ of dichloromethane. Column chromatography (30:70 EtOAc:hex) 
to give 16 as white crystalline solid $(0.95 \mathrm{~g}, 73 \%)$. Mp $92-94^{\circ} \mathrm{C} .{ }^{1} \mathrm{H}-\mathrm{NMR}\left(400 \mathrm{MHz}, \mathrm{CDCl}_{3}\right)$ : $\delta 10.79(\mathrm{~s}, 1 \mathrm{H}, \mathrm{NH}), 9.03(\mathrm{~s}, 1 \mathrm{H}, \mathrm{NH}), 7.90-7.88(\mathrm{~d}, 1 \mathrm{H}, J=8.0 \mathrm{~Hz}), 7.80-7.78(\mathrm{~d}, 1 \mathrm{H}, J=7.8$ Hz), 7.29-7.25 (t, 1H, J = 8.0 Hz), $5.00(\mathrm{~s}, 1 \mathrm{H}), 4.89(\mathrm{~s}, 1 \mathrm{H}), 3.86(\mathrm{~s}, 3 \mathrm{H}), 3.44-3.40(\mathrm{~m}, 4 \mathrm{H})$, 3.32-3.26 (m, 4H). ${ }^{13} \mathrm{C}-\mathrm{NMR}\left(100.6 \mathrm{MHz}, \mathrm{CDCl}_{3}\right):{ }^{\delta}$ 170.1, 169.5, 167.2, 132.3, 131.4, 130.9, 128.2, 126.1, 122.9, 54.1, 54.0, 52.7, 39.4, 39.3, IR (KBr): 3237, 2926, 1718, 1676, 1646, 1508 , 1301, 1142, $758 \mathrm{~cm}^{-1}$. Elemental Analysis: Calcd for $\mathrm{C}_{16} \mathrm{H}_{18} \mathrm{~N}_{2} \mathrm{O}_{4} \mathrm{~S}_{4}: \mathrm{C}, 44.63 ; \mathrm{H}, 4.21 ; \mathrm{N}, 6.51$. Found: $\mathrm{C}, 44.68 ; \mathrm{H}, 4.18 ; \mathrm{N}, 6.38$. Structure was confirmed by single crystals data.

2,6-Bis-(2-phenylsulfanyl-acetylamino)-benzoic acid methyl ester (17): In the manner<smiles>COC(=O)c1c(NC(=O)CSc2ccccc2)cccc1NC(=O)CSc1ccccc1</smiles>
described above, 2,6-bis-amino-benzoic acid methyl ester (5) $(0.25$ $\mathrm{g}, 1.5 \mathrm{mmol})$ was treated with thiophenyl acetyl chloride $(0.618 \mathrm{~g}$, $3.3 \mathrm{mmol})$ in presence of $480 \mathrm{mg}(6.0 \mathrm{mmol})$ of pyridine at $0^{\circ} \mathrm{C}$ in $20 \mathrm{~mL}$ of dichloromethane. Column chromatography (30:70 EtOAc:hex) to give $\mathbf{1 7}$ as white crystalline solid (0.6 g, 85\%). Mp

120-122 ${ }^{\circ} \mathrm{C} . \quad{ }^{1} \mathrm{H}-\mathrm{NMR}\left(400 \mathrm{MHz}, \mathrm{CDCl}_{3}\right):{ }^{\delta} 10.79$ (s, 2H, NH), 8.16-8.14 (d, 2H, $\left.J=8.3 \mathrm{~Hz}\right)$, 7.51-7.47 (t, 1H, $J=8.3 \mathrm{~Hz}), 7.32-7.28(\mathrm{~m}, 8 \mathrm{H}), 7.24-7.22(\mathrm{~m}, 2 \mathrm{H}), 3.79(\mathrm{~s}, 4 \mathrm{H}), 3.76(\mathrm{~s}, 3 \mathrm{H})$. ${ }^{13} \mathrm{C}-\mathrm{NMR}\left(100.6 \mathrm{MHz}, \mathrm{CDCl}_{3}\right):{ }^{\delta} 166.8,166.7,138.5,134.4,133.4,129.3,128.2,126.8,117.9$, 109.5, 52.9, 38.9. IR (KBr): 3338, 3290, 2950, 1714, 1685, 1583, 1218, 1116, $735 \mathrm{~cm}^{-1}$. Elemental Analysis: Calcd for $\mathrm{C}_{24} \mathrm{H}_{22} \mathrm{~N}_{2} \mathrm{O}_{4} \mathrm{~S}_{2}$ : C, 61.78; H, 4.75; N, 6.00. Found: C, 61.91; H, 4.47; N, 5.96. Structure was confirmed by by single crystals data.

2,3-Bis-(2-phenylsulfanyl-acetylamino)-benzoic acid methyl ester (18): In the manner<smiles>COC(=O)c1cccc(NC(=O)Cc2ccccc2)c1NC(=O)Cc1ccccc1</smiles>

18 described above, 2,3-bis-amino-benzoic acid methyl ester (10) $(0.25 \mathrm{~g}, 1.5$ mmol) was treated with thiophenyl acetyl chloride $(0.618 \mathrm{~g}, 3.3 \mathrm{mmol}$ in presence of $480 \mathrm{mg}(6.0 \mathrm{mmol})$ of pyridine at $0^{\circ} \mathrm{C}$ in $20 \mathrm{~mL}$ of 
dichloromethane. Column chromatography (30:70 EtOAc:hex) to give $\mathbf{1 8}$ as white solid (0.58 g, 85\%). Mp 100-102 ${ }^{\circ} \mathrm{C} .{ }^{1} \mathrm{H}-\mathrm{NMR}\left(400 \mathrm{MHz}, \mathrm{CDCl}_{3}\right):{ }^{\delta} 10.76$ (s, $\left.1 \mathrm{H}, \mathrm{NH}\right), 9.40$ (s, 1H, NH), 7.85-7.79 (m, 2H), 7.36-7.20 (m, 11H), $3.77(\mathrm{~s}, 3 \mathrm{H}), 3.69$ (s, 2H), $3.53(\mathrm{~s}, 2 \mathrm{H}) .{ }^{13} \mathrm{C}-\mathrm{NMR}(100.6$ $\left.\mathrm{MHz}, \mathrm{CDCl}_{3}\right):{ }^{\delta} 168.8,167.1,167.0,135.1,134.7,132.0,131.7,131.1,129.2,129.1,128.4$, 128.2, 126.7, 126.5, 125.9, 123.0, 52.5, 38.2, 38.0. IR (KBr): 3276, 3239, 3056, 2957, 1706, 1670, 1495, 1306, 1151, $787 \mathrm{~cm}^{-1}$. Elemental Analysis: Calcd for $\mathrm{C}_{24} \mathrm{H}_{22} \mathrm{~N}_{2} \mathrm{O}_{4} \mathrm{~S}_{2}: \mathrm{C}, 61.78 ; \mathrm{H}$, 4.75; N, 6.00. Found: C, 61.90; H, 4.77; N, 5.90.

2,6-Bis-(2-benzyloxyimino-acetylamino)-benzoic acid methyl ester (19): 2,6-Bis-amino-<smiles>CCCOCC(=O)Nc1cccc(NC(=O)/C=N/OC(=O)/C=N/OCc2ccccc2)c1C(=O)OC</smilesbenzoic acid methyl ester (5) (0.25 g, $1.5 \mathrm{mmol})$ was dissolved in dichloromethane $(20 \mathrm{~mL})$ and cooled in an ice bath. Triethylamine (365 $\mathrm{mg}, 3.6 \mathrm{mmol}$ ) was added, and the solution was stirred for 15 minutes. $\alpha$-benzyloximino acid chloride ${ }^{30}$ (650 mg, $3.2 \mathrm{mmol})$ was dissolved in dichloromethane $(5 \mathrm{~mL})$ and the solution was added dropwise over a period of 15 minutes. The reaction mixture was stirred in the ice bath for $1 \mathrm{~h}$ and stirred at room temperature overnight, and reaction mixture was quenched with aqueous solution of $\mathrm{HCl}$ and extracted with dichloromethane (50 $\mathrm{mL} \mathrm{X} \mathrm{2),} \mathrm{and}$ the organic layer was washed with water $(100 \mathrm{~mL})$, saturated sodium bicarbonate solution (100 $\mathrm{mL})$, water $(100 \mathrm{~mL})$, brine $(100 \mathrm{~mL})$, dried over anhydrous $\mathrm{MgSO}_{4}$ and filtered. All volatiles were removed and the residue was purified using column chromatography (15:85 EtOAc:hex) to give 19 as white solid. $(0.4 \mathrm{~g}, 55 \%) . \mathrm{Mp} 108-110^{\circ} \mathrm{C} .{ }^{1} \mathrm{H}-\mathrm{NMR}\left(400 \mathrm{MHz}, \mathrm{CDCl}_{3}\right):{ }^{\delta} 10.87$ (s, 2H, NH), 8.30-8.28 (d, 2H, J=8.4 Hz), $7.59(\mathrm{~s}, 2 \mathrm{H}), 7.54-7.50(\mathrm{t}, 1 \mathrm{H}, J=8.3 \mathrm{~Hz}), 7.46-7.38(\mathrm{~m}$, 10H), 5.31 (s, 4H), 3.85 (s, 3H). ${ }^{13} \mathrm{C}-\mathrm{NMR}\left(100.6 \mathrm{MHz}, \mathrm{CDCl}_{3}\right):{ }^{\delta}$ 167.4, 160.2, 144.5, 139.3, 136.6, 134.3, 129.0, 128.9, 128.8, 117.8, 108.4, 78.1, 53.0. IR (KBr): 3357, 3031, 2952, 1695, 
1676, 1585, 1500, 1407, 1270, $916 \mathrm{~cm}^{-1}$. Elemental Analysis: Calcd for $\mathrm{C}_{26} \mathrm{H}_{24} \mathrm{~N}_{4} \mathrm{O}_{6}$ : C, 63.93;

H, 4.95; N, 11.48. Found: C, 64.02; H, 4.94; N, 11.32 and eluted with (20:80 EtOAc:hex) to give $\mathbf{2 0}$ as white solid (90 $\mathrm{mg}, 18 \%) . \mathrm{Mp} 90-92^{\circ} \mathrm{C}$.

2-Amino-6-(2-benzyloxyimino-acetylamino)-benzoic acid methyl ester (20): In the manner<smiles>COC(=O)c1c(N)cccc1NC(=O)/C=N/OCc1ccccc1</smiles>
described above, 2,6-bis-amino-benzoic acid methyl ester (5) (0.25 g, 1.5 mmol) was treated with $\alpha$-benzyloximino acid chloride ${ }^{30}$ (326 mg, 1.6 $\mathrm{mmol})$ in presence of $0.25 \mathrm{~mL},(190 \mathrm{mg}, 1.8 \mathrm{mmol})$ of triethylamine at $0^{\circ} \mathrm{C}$ in $20 \mathrm{~mL}$ of dichloromethane. Column chromatography (20:80 EtOAc:hex) to give 20 as white solid $(0.3 \mathrm{~g}, 62 \%)$. Mp 90-92 ${ }^{\circ} \mathrm{C} .{ }^{1} \mathrm{H}-\mathrm{NMR}\left(400 \mathrm{MHz}, \mathrm{CDCl}_{3}\right){ }^{\circ} 11.21$ (br-s, 1H, NH), 7.82-7.80 (d, 1H, J = 8.2 Hz), $7.46(\mathrm{~s}, 1 \mathrm{H}), 7.33-7.23(\mathrm{~m}, 5 \mathrm{H}), 7.08-7.06(\mathrm{t}, 1 \mathrm{H}, J=8.2$ Hz), 6.31-6.29 (d, 1H, J = 8.2 Hz), 5.42 (br-s, 2H, NH2), 5.18 (s, 2H), 3.75 (s, 3H). ${ }^{13} \mathrm{C}-\mathrm{NMR}$ (100.6 MHz, $\left.\mathrm{CDCl}_{3}\right){ }^{\delta} 168.1,159.6,150.7,144.5,139.6,136.2,133.9,128.4,128.3,128.2$, 112.6, 109.6, 100.9, 77.4, 51.7. IR (KBr): 3456, 3317, 3023, 1683, 1618, 1543, 1462, 1234, 922 $\mathrm{cm}^{-1}$. Elemental Analysis: Calcd for $\mathrm{C}_{17} \mathrm{H}_{17} \mathrm{~N}_{3} \mathrm{O}_{4}$ : C, 62.38; H, 5.23; N, 12.84. Found: C, 62.65; H, 5.75; N, 12.89. and eluted with (15:85 EtOAc:hex) to give diester product 19 as white solid (40 mg, 5\%). Mp 108-110 $\mathrm{C}$.

\section{2,2-Dimethyl-2,3-dihydro-1H-benzoimidazole-4-carboxylic acid methyl ester (21):}

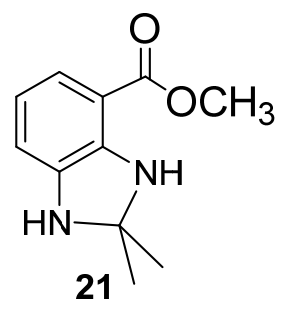

2,3-Bis-amino-benzoic acid methyl ester (10) $(0.5 \mathrm{~g}, 3.0 \mathrm{mmol})$ and 2,2'dimethoxypropane $(5.0 \mathrm{~mL})$ was stirred in presence of catalytic amount $(100$ $\mathrm{mg}$ ) of para-toulenesulfonic acid at room temperature for $16 \mathrm{~h}$. Reaction mixture on concentrated gives the residue, which on dilution with dichloromethane $(100 \mathrm{~mL})$. Separate the organic layer and wash with saturated sodium bicarbonate solution $(100 \mathrm{~mL})$, water $(100 \mathrm{~mL})$, and brine solution $(100 \mathrm{~mL})$ and dried over 
$\mathrm{MgSO}_{4}(5.0 \mathrm{~g})$ and filter. Concentrate the filtrate and the residue on column chromatography (20:80 EtOAc:hex) to give 22 as residual oil $(0.3 \mathrm{~g}, 47 \%) .{ }^{1} \mathrm{H}-\mathrm{NMR}\left(400 \mathrm{MHz}, \mathrm{CDCl}_{3}\right){ }^{\circ}{ }^{\overline{7}} 7.00-$ 6.98 (d, 1H, $J=7.2 \mathrm{~Hz}), 6.36-6.33$ (t, 2H, $J=7.5 \mathrm{~Hz}$ ), 5.93 (br-s, 1H, NH), 3.86 (br-s, 1H, NH), $3.73\left(\mathrm{~s}, 3 \mathrm{H}, \mathrm{COOCH}_{3}\right) .{ }^{13} \mathrm{C}-\mathrm{NMR}\left(100.6 \mathrm{MHz}, \mathrm{CDCl}_{3}\right):{ }^{\delta} 167.9,143.3,139.6,118.8,116.6$, 110.2, 106.3, 79.0, 51.1, 30.0. IR (KBr): 3372, 2975, 1687, 1573, 1485, $1257 \mathrm{~cm}^{-1}$.

2,3-Bis-(2-benzyloxyimino-acetylamino)-benzoic acid methyl ester (22): The compound 22,

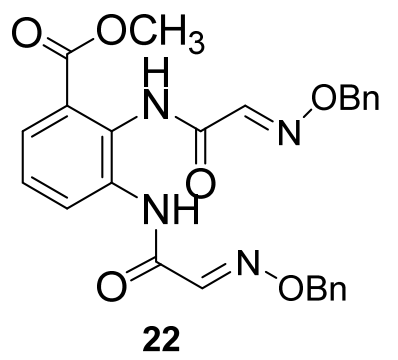

$(0.5 \mathrm{~g}, 2.4 \mathrm{mmol})$ was dissolved in THF $(10 \mathrm{~mL})$ and cooled in an ice bath. Sodium hydride (138 $\mathrm{mg}, 5.7 \mathrm{mmol})$ was added and the mixture was stirred 15 minutes. $\alpha$-benzyloximino acid chloride ${ }^{30}$ solution (1.04 $\mathrm{g}, 5.2 \mathrm{mmol})$ in dry THF $(10 \mathrm{~mL})$ was added to the reaction mixture and stirred at room temperature for $24 \mathrm{~h}$. The THF was removed and the residue was quenched by the addition of $10 \%$ aqueous $\mathrm{HCl}(100 \mathrm{~mL})$ and stir for $3 \mathrm{~h}$. The aqueous solution was extracted with ethyl acetate $(100 \mathrm{~mL})$, and the organic layer was washed with water $(100 \mathrm{~mL})$, saturated sodium bicarbonate solution $(100 \mathrm{~mL})$, water $(100 \mathrm{~mL})$, brine (100 mL), and dried over anhydrous $\mathrm{MgSO}_{4}$. After filtration and concentration, the residue was purified by column chromatography (20:80 EtOAc:hex) to give $\mathbf{2 3}$ as white solid ( $0.9 \mathrm{~g}, \mathbf{7 8 \%}$ ). Mp 150-152 ${ }^{\circ} \mathrm{C} .{ }^{1} \mathrm{H}-\mathrm{NMR}\left(400 \mathrm{MHz}, \mathrm{CDCl}_{3}\right):{ }^{\delta} 10.95$ (s, $\left.1 \mathrm{H}, \mathrm{NH}\right), 9.06$ (s, $\left.1 \mathrm{H}, \mathrm{NH}\right), 8.05-8.03$ $(\mathrm{d}, 1 \mathrm{H}, J=7.9 \mathrm{~Hz}), 7.91-7.89(\mathrm{~d}, 1 \mathrm{H}, J=7.8 \mathrm{~Hz}), 7.56(\mathrm{~s}, 1 \mathrm{H}), 7.54(\mathrm{~s}, 1 \mathrm{H}), 7,52-7.50(\mathrm{t}, 1 \mathrm{H}, J=$ 6.6 Hz), 7.45-7.35 (m, 10H), $5.36(\mathrm{~s}, 2 \mathrm{H}), 5.24(\mathrm{~s}, 2 \mathrm{H}), 3.94(\mathrm{~s}, 3 \mathrm{H}) .{ }^{13} \mathrm{C}-\mathrm{NMR}(100.6 \mathrm{MHz}$, $\left.\mathrm{CDCl}_{3}\right){ }^{\delta} 167.2,160.8,160.3,143.3,142.6,136.3,135.9,131.6,131.3,130.8,129.0,128.7$, 128.6, 128.5, 128.4, 128.1, 125.8, 122.7, 78.3, 77.8, 52.5. IR (KBr): 3448, 3246, 1697, 1681, 1534, 1000, $754 \mathrm{~cm}^{-1}$. Elemental Analysis: Calcd for $\mathrm{C}_{26} \mathrm{H}_{24} \mathrm{~N}_{4} \mathrm{O} 6$ : C, 63.93; H, 4.95; N, 11.48 . Found: C, 63.94; H, 4.80; N, 11.37 . 
<smiles>COC(=O)c1c(NC(=O)/C=N/OCc2ccccc2)cccc1NC(=O)C1SCCS1</smiles>

Column chromatography (30:70 EtOAc:hex) to give 21 as pale yellow solid (225 mg, 64\%). Mp 170-172 ${ }^{\circ} \mathrm{C} .{ }^{1} \mathrm{H}-\mathrm{NMR}\left(400 \mathrm{MHz}, \mathrm{CDCl}_{3}\right):{ }^{\delta} 10.91$ (s, 1H, NH), 10.69 (s, 1H, NH), 8.27-8.25 (d, 1H, J=7.9 Hz), 8.20-8.18 d, 1H, J= 7.6 Hz), 7.59 (s, 1H), 7.52-7.50 (t, 1H, J=7.7 Hz), $7.42(\mathrm{~m}, 5 \mathrm{H}), 5.31(\mathrm{~s}, 2 \mathrm{H}), 5.03(\mathrm{~s}, 1 \mathrm{H}), 3.93(\mathrm{~s}, 3 \mathrm{H}), 3.43-3.40(\mathrm{~m}$, 4H). ${ }^{13} \mathrm{C}-\mathrm{NMR}\left(100.6 \mathrm{MHz}, \mathrm{CDCl}_{3}\right):{ }^{\delta} 169.1,167.5,144.6,139.7,138.9,136.6,134.2,129.0$, 128.9, 128.6, 118.1, 118.1, 109.1, 78.1, 55.1, 53.2, 39.6. IR (KBr): 3448, 3290, 2948, 1695, $1684,1659,1534,1500,1467,1287,806 \mathrm{~cm}^{-1}$. Elemental Analysis: Calcd for $\mathrm{C}_{21} \mathrm{H}_{21} \mathrm{~N}_{3} \mathrm{O}_{5} \mathrm{~S}_{2}$ : C, 54.89; H, 4.61; N, 9.14. Found: C, 54.13; H, 4.45; N, 8.88.

Dimethyl 6,6'-(carbonylbis(azanediyl))-bis(2-nitrobenzoate) (24): Dissolved compound 3 (2.5<smiles>COC(=O)c1c(NC(=O)Nc2cccc([N+](=O)[O-])c2C(=O)OC)cccc1[N+](=O)[O-]</smiles>

24 $\mathrm{g}, 10.0 \mathrm{mmol}$ ) in aqueous glacial acetic acid $10 \mathrm{~mL} / 5 \mathrm{~mL})$ and heated at $110^{\circ} \mathrm{C}$ until no more nitrogen gas evolved $(2 \mathrm{~h})$. Poured the reaction mixture into crushed ice (100 g) and stirred for 30 minutes to precipitate the product. Filtered and washed well with water $(250 \mathrm{~mL})$. After dry obtained 1.9 of essentially pure product in $91 \%$ yield. ${ }^{1} \mathrm{H}-\mathrm{NMR}$ (DMSO-d6, 400): ${ }^{\delta} 9.10$ (s, 1H, NH urea), 8.12-8.10 (d, 1H, J= 7.6 Hz, CH alpha to Nitro), 7.87-7.85 (d, $1 \mathrm{H}, J=7.6 \mathrm{~Hz}, \mathrm{CH}$ alpha to urea), 7.73-7.71 $(1 \mathrm{H}, J=7.4 \mathrm{~Hz}, \mathrm{CH}$ meta), $3.83\left(\mathrm{~S}, 3 \mathrm{H}, \mathrm{COOCH}_{3}\right) .{ }^{1} \mathrm{H}-\mathrm{NMR}\left(\mathrm{CDCl}_{3}, 400 \mathrm{MHz}\right):{ }^{\delta} 9.02$ (s, 1H, NH urea), 8.51-8.49 (d, 1H, J = 7.6 Hz, CH alpha to Nitro), 7.63-7.54 (m, 2H, alpha to urea, $\mathrm{CH}$ meta), 3.93 (S, 3H, 
$\left.\mathrm{COOCH}_{3}\right) .{ }^{13} \mathrm{C}-\mathrm{NMR}$ (DMSO-d6, $\left.400 \mathrm{MHz}\right){ }^{\delta} 165.4$ (CO, ester), 153.3 (CO, Urea), 148.3 (C-

Nitro), 138.0 (C-NH-Urea), $132.2(\mathbf{C H}$, alpha to Nitro), $130.4(\mathbf{C H}$ alpha to urea), 120,6 (CCOOMe), 120.2 ( $\mathbf{C H}$ meta), $54.3\left(\mathrm{CH}_{3}, \mathrm{COOCH}_{3}\right)$.

\section{Reference:}

1. $\quad$ Bu, X.-H.; Hou, W.-F.; Du, M.; Chen, W.; Zhang, R.-H., Varying the Frameworks of Novel Silver(I) Coordination Polymers with Thioethers by Altering the Backbone or Terminal Groups of Ligands. Cryst. Growth Des. 2002, 2 (4), 303-307.

2. Bu, X.-H.; Chen, W.; Hou, W.-F.; Du, M.; Zhang, R.-H.; Brisse, F., Controlling the Framework Formation of Silver(I) Coordination Polymers with 1,4-Bis(phenylthio)butane by Varying the Solvents, Metal-to-Ligand Ratio, and Counteranions. Inorg. Chem. 2002, 41 (13), 3477-3482.

3. Zellner, C. N.; Dougherty, G., Chemiluminescence of phthalhydrazide derivatives. J. Am. Chem. Soc. 1937, 59, 2580-3.

4. Wamser, C. C.; Phillips, R. B., Chemiluminescent oxidations of 4- and 7-aminophthalide. The Journal of Organic Chemistry 1976, 41 (17), 2929-2931.

5. Wildes, P. D.; White, E. H., Differences between excited states produced chemically and photochemically. Ion pairs of excited states derived from luminol. Journal of the American Chemical Society 1973, 95 (8), 2610-2617.

6. Aramu, F.; Maxia, V.; Serra, M.; Spano, G., Thermoluminescence of hydroxy and aminobenzoic acids. Journal of Luminescence 1972, 5 (6), 439-448.

7. $\quad$ de Silva, A. P.; Dixon, I. M.; Gunaratne, H. Q. N.; Gunnlaugsson, T.; Maxwell, P. R. S.; Rice, T. E., Integration of Logic Functions and Sequential Operation of Gates at the MolecularScale. Journal of the American Chemical Society 1999, 121 (6), 1393-1394.

8. Hardy, G. E.; Kaska, W. C.; Chandra, B. P.; Zink, J. I., Triboluminescence-structure relationships in polymorphs of hexaphenylcarbodiphosphorane and anthranilic acid, molecular crystals, and salts. Journal of the American Chemical Society 1981, 103 (5), 1074-1079.

9. Choi, M. S.; Lee, J. H.; Kim, D. W.; Lee, T. S., New conjugated polymers comprising ortho-phenylazonaphthols: Synthesis and chromogenic behaviors. Journal of Polymer Science Part A: Polymer Chemistry 2007, 45 (19), 4430-4440.

10. Holbrey, J. D.; Reichert, W. M.; Tkatchenko, I.; Bouajila, E.; Walter, O.; Tommasi, I.; Rogers, R. D., 1,3-Dimethylimidazolium-2-carboxylate: the unexpected synthesis of an ionic liquid precursor and carbene-CO2 adduct. Chemical Communications 2003, (1), 28-29.

11. Huntress, E. H.; Gladding, J. V. K., The Synthesis of Aminobenzoyleneureas and of Dihydroxyquinoxalines Isomeric with "Luminol". Journal of the American Chemical Society 1942, 64 (11), 2644-2649.

12. Makishima, A., Preface. In Biochemistry for Materials Science, Makishima, A., Ed. Elsevier: 2019; pp xxvii-xxviii.

13. López Rubio, A.; Gómez-Mascaraque, L. G.; Fabra, M. J.; Martínez Sanz, M., Chapter 1 - Nanomaterials for Food Applications: General Introduction and Overview of the Book. In Nanomaterials for Food Applications, López Rubio, A.; Fabra Rovira, M. J.; martínez Sanz, M.; Gómez-Mascaraque, L. G., Eds. Elsevier: 2019; pp 1-9. 
14. Sengupta, P.; Zhang, H.; Son, D. Y., Spacer Flexibility in Bis(pyridyl) Ligands: Chelating Organosilicon Pyridylethynyl Ligands. Inorganic Chemistry 2004, 43 (6), 1828-1830.

15. Kalra, M. K.; Zhang, H.; Son, D. Y., Helical metallopolymers from the coordination of new bis(pyridyl)terephthalate ligands with silver(I). Inorganic Chemistry Communications 2004, 7 (9), 1019-1022.

16. Gondi, S. R.; Son, D. Y., Synthesis of N,N'-bis(2-thiazolinyl)-, N,N'-bis(2-thiazolyl)-, and N,N'-bis(2-pyrimidinyl)-benzenedicarboxamides. Synth. Commun. 2004, 34 (17), 30613072 .

17. Gondi, S. R.; Son, D. Y., Mono-, bis-, and tris-1,3-dithiolane aromatic derivatives by esterification and amidation reactions. J. Sulfur Chem. 2005, 26 (1), 13-19.

18. Gondi, S. R.; Son, D., Cholane Derivatives with Potential Ligating Groups at the 3- and 24-Positions. Synth. Commun. 2006, 36 (10), 1317-1331.

19. Gondi, S. R.; Son, D. Y., Synthesis of (hetaryl)alkylamines from the reactions of 2aminopyrimidine, 2-aminothiazole, and 2-aminothiazoline with benzyl bromide and xylylene dibromides. Synth. Commun. 2008, 38 (3), 401-410.

20. Zhang, H.; Gondi, S. R.; Son, D. Y., Poly[bis $(\mu 3-$ benzyloxyiminoacetato)nitratotrisilver(I)]. Acta Crystallogr., Sect. E: Struct. Rep. Online 2006, 62 (7), m1613-m1615.

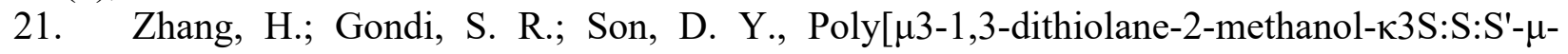
nitrato-silver(I)]. Acta Crystallogr., Sect. E: Struct. Rep. Online 2006, 62 (11), m3086-m3088.

22. Herscheid, J. D. M.; Colstee, J. H.; Ottenheijm, H. C. J., 1,4-Dihydroxy-2,5dioxopiperazines from activated N-hydroxyamino acids. The Journal of Organic Chemistry 1981, 46 (16), 3346-3348.

23. Papadopoulos, E. P.; Torres, C. D., Convenient preparation of N-substituted 2-amino-4H3,1-benzoxazin-4-ones and 3-substituted 2,4(1H,3H)-quinazolinediones. Journal of Heterocyclic Chemistry 1982, 19 (2), 269-272.

24. Aziane, D.; Amzazi, S.; Bakri, Y.; Essassi, E. M.; Akssira, M.; Benjouad, A., Synthesis of new quinazolinediones and evaluation of their effect against the human immunodeficiency virus. J. Maroc. Chim. Heterocycl. 2003, 2 (1), 57-63.

25. Sweeting, L. M., Triboluminescence with and without Air. Chemistry of Materials 2001, 13 (3), 854-870.

26. Funk, P.; Motyka, K.; Džubák, P.; Znojek, P.; Gurská, S.; Kusz, J.; McMaster, C.; Hajdúch, M.; Soural, M., Preparation of 2-phenyl-3-hydroxyquinoline-4(1H)-one-5carboxamides as potential anticancer and fluorescence agents. RSC Advances 2015, 5 (60), 48861-48867.

27. Melnik, M.; Mikus, P., Crystallographic and structural analysis of heterometallic FeAg complexes. Int. Res. J. Pure Appl. Chem. 2015, 5 (2), 1-17, 17 pp.

28. Garcia-Rodriguez, J.; Mendiratta, S.; White, M. A.; Xie, X.-S.; De Brabander, J. K., Synthesis and structure-activity studies of the V-ATPase inhibitor saliphenylhalamide (SaliPhe) and simplified analogs. Bioorg. Med. Chem. Lett. 2015, 25 (20), 4393-4398.

29. Ilangovan, A.; Sakthivel, P.; Sakthivel, P., Green and practical transition metal-free onepot conversion of substituted benzoic acids to anilines using tosyl azide. Org. Chem. Front. 2016, 3 (12), 1680-1685.

30. Reddy, G. S.; Chen, H.-Y.; Chang, I. J., Cysteine-specific blue fluorescence probe. J. Chin. Chem. Soc. (Taipei, Taiwan) 2006, 53 (6), 1303-1308. 
31. Aziane, D.; Soukri, M.; El Hakmaoui, A.; Lazar, S.; Akssira, M.; Essassi, E. M.; Guillaumet, G., A convenient synthesis of 5- and 8-nitroquinazoline-2,4-dione derivatives. Journal of Heterocyclic Chemistry 2002, 39 (2), 271-276.

32. Bhatt, M. V.; El Ashry, S. H.; Somayaji, V., Aspects of tautomerism. Part X. Neighboring group effects on the structure and reactivity patterns of acid chlorides. Indian J. Chem., Sect. B 1980, 19B (6), 473-86.

33. Kubo, K.; Kohara, Y.; Imamiya, E.; Sugiura, Y.; Inada, Y.; Furukawa, Y.; Nishikawa, K.; Naka, T., Nonpeptide angiotensin II receptor antagonists. Synthesis and biological activity of benzimidazolecarboxylic acids. Journal of Medicinal Chemistry 1993, 36 (15), 2182-2195.

34. Endo, H.; Wempe, M. F.; Peyron, J.-F.; Anzai, N.; Jutabha, P. Anti-malignant tumor agent composition comprising LAT1 inhibitor in combination with other active agent. WO2015173970A1, 2015. 